\title{
A DEGREE CONDITION FOR THE EXISTENCE OF $k$-FACTORS WITH PRESCRIBED PROPERTIES
}

\author{
CHANGPING WANG
}

Received 27 July 2004 and in revised form 26 December 2004

Let $k$ be an integer such that $k \geq 3$, and let $G$ be a 2-connected graph of order $n$ with $n \geq 4 k+1, k n$ even, and minimum degree at least $k+1$. We prove that if the maximum degree of each pair of nonadjacent vertices is at least $n / 2$, then $G$ has a $k$-factor excluding any given edge. The result of Nishimura (1992) is improved.

\section{Introduction and result}

We consider only finite undirected graphs without loops or multiple edges. Let $G$ be a graph with vertex set $V(G)$ and edge set $E(G)$. For a vertex $x \in V(G)$, we write $N_{G}(v)$ for the set of vertices of $V(G)$ adjacent to $v, N_{G}[v]$ for $N_{G}(v) \cup\{v\}$, and $d_{G}(v)=\left|N_{G}(v)\right|$ for the degree of $v$ in $G$. If $S$ and $T$ are disjoint subsets of $V(G)$, then $e_{G}(S, T)$ denotes the number of edges that join $S$ and $T$, and $G-S$ denotes the subgraph of $G$ obtained from $G$ by deleting the vertices in $S$ together with the edges incident with them. A $k$-factor of $G$ is a spanning subgraph $F$ of $G$ such that $d_{F}(x)=k$ for every $x \in V(F)$. If $G$ and $H$ are disjoint graphs, then the join and the union are denoted by $G+H$ and $G \cup H$, respectively. Other terminology and notation not defined here can be found in [1].

The following theorems of $k$-factors in terms of degree conditions are known.

Theorem 1.1 (Nishimura [4]). Let $k$ be an integer such that $k \geq 3$, and let $G$ be a connected graph of order $n$ with $n \geq 4 k-3, k n$ even, and minimum degree at least $k$. Suppose that $\max \left(d_{G}(u), d_{G}(v)\right) \geq n / 2$ for each pair of nonadjacent vertices $u$, $v$ of $V(G)$. Then $G$ has a $k$-factor.

Theorem 1.2 (Iida and Nishimura [3]). Let $k$ be a positive integer, and let $G$ be a graph of order $n$ with $n \geq 4 k-5, k n$ even, and minimum degree at least $k$. If the degree sum of each pair of nonadjacent vertices is at least $n$, then $G$ has a $k$-factor.

Theorem 1.3 (Egawa and Enomoto [2]). Let $k$ be a positive integer, and let $G$ be a graph of order $n$ with $n \geq 4 k-5, k n$ even, and minimum degree at least $n / 2$. Then $G$ has a $k$-factor.

The main result of this paper is the following theorem.

Theorem 1.4. Let $k$ be an integer such that $k \geq 3$, and let $G$ be a 2-connected graph of order $n$ with $n \geq 4 k+1, k n$ even, and minimum degree at least $k+1$. Suppose that 
$\max \left(d_{G}(u), d_{G}(v)\right) \geq n / 2$ for each pair of nonadjacent vertices $u$, $v$ of $V(G)$. Then for any $e \in E(G), G-e$ has a $k$-factor.

The assumptions in Theorem 1.4 cannot be weakened any further. We discuss them in the last section.

\section{Proof of Theorem 1.4}

In order to prove Theorem 1.4, the following definitions are needed.

Let $G$ be a graph, and $S, T \subseteq V(G)$ with $S \cap T=\varnothing$. For an integer $k \geq 1$, a component $C$ of $G-(S \cup T)$ is called a $k$-odd component or $k$-even component according to whether $k|V(C)|+e_{G}(V(C), T)$ is odd or even. Assume that $e$ is a cut edge of $G-(S \bigcup T)$ and $C(e)$ is the component of $G-(S \cup T)$ which contains $e$. We say that $e$ is a $k$-odd cut edge or $k$-even cut edge according to parity, that is, whether both components of $C(e)-e$ are $k$-odd components or $k$-even components of $(G-e)-(S \bigcup T)$. (Note that $C(e)$ must be a $k$-even component of $G-(S \cup T)$ in both cases.) We write

$$
\delta_{G}(S, T)=k|S|+\sum_{x \in T} d_{G-S}(x)-k|T|-h_{G}(S, T)
$$

where $h_{G}(S, T)$ is the number of $k$-odd components of $G-(S \cup T)$.

Lemma 2.1 (Tutte [5]). Let $G$ be a graph and $k$ a positive integer. For all disjoint subsets $S$ and $T$ of $V(G), G$ has a $k$-factor if and only if

(i) $\delta_{G}(S, T) \geq 0$,

(ii) $\delta_{G}(S, T) \equiv k n(\bmod 2)$.

Lemma 2.2. A graph $G$ has a $k$-factor excluding any given edge if and only if $\delta_{G}(S, T) \geq$ $\varepsilon(S, T)$ for all disjoint subsets $S$ and $T$ of $V(G)$, where $\varepsilon(S, T)=2$ if $G[T]$ has an edge, or $G-(S \bigcup T)$ has a $k$-odd cut edge, or $G-(S \bigcup T)$ has a k-even component $C$ such that $e_{G}(V(C), T) \geq 1$; otherwise, $\varepsilon(S, T)=0$.

Proof. A graph $G$ has a $k$-factor excluding any given edge if and only if $G-e$ has a $k$-factor for every $e \in E(G)$. By Lemma 2.1, $G-e$ has a $k$-factor if and only if $\delta_{G-e}(S, T) \geq 0$ for all disjoint subsets $S$ and $T$ of $V(G)$. So, a graph $G$ has a $k$-factor excluding any given edge if and only if for all disjoint subsets $S$ and $T$ of $V(G)$,

$$
\min _{e \in E(G)} \delta_{G-e}(S, T) \geq 0 .
$$

Note that $\delta_{G}(S, T)=k|S|+\sum_{x \in T} d_{G-S}(x)-k|T|-h_{G}(S, T)$ and $\delta_{G-e}(S, T)=k|S|+$ $\sum_{x \in T} d_{G-e-S}(x)-k|T|-h_{G-e}(S, T)$. By the definition of $\varepsilon(S, T)$, we know that

$$
\begin{aligned}
\varepsilon(S, T) & =\max _{e \in E(G)}\left[\left(\sum_{x \in T} d_{G-S}(x)-\sum_{x \in T} d_{G-e-S}(x)\right)+\left(h_{G-e}(S, T)-h_{G}(S, T)\right)\right] \\
& =\max _{e \in E(G)}\left(\delta_{G}(S, T)-\delta_{G-e}(S, T)\right) \\
& =\delta_{G}(S, T)-\min _{e \in E(G)} \delta_{G-e}(S, T) .
\end{aligned}
$$


So,

$$
\min _{e \in E(G)} \delta_{G-e}(S, T)=\delta_{G}(S, T)-\varepsilon(S, T)
$$

which completes the proof.

Lemma 2.3. Let $G$ be a graph $G$ and $k \geq 1$. Assume that there exists a real number $\theta$ and disjoint subsets $S$ and $T$ of $V(G)$ satisfying

(i) $\delta_{G}(S, T)<\theta$,

(ii) $|S \cup T|$ is as large as possible.

Then $d_{G-S}(u) \geq k+1$ and $e_{G}(u, T) \leq k-1$ for all $u \in V(G)-(S \cup T)$. Moreover, the order of each component of $G-(S \cup T)$ is at least 3.

Proof. If there is $u^{*} \in V(G)-(S \bigcup T)$ such that $d_{G-S}\left(u^{*}\right) \leq k$. Set $S^{*}=S, T^{*}=T \bigcup\left\{u^{*}\right\}$, we have

$$
\begin{aligned}
\delta_{G}\left(S^{*}, T^{*}\right) & =k\left|S^{*}\right|+\sum_{x \in T^{*}} d_{G-S}(x)-k\left|T^{*}\right|-h_{G}\left(S^{*}, T^{*}\right) \\
& =k|S|+\sum_{x \in T} d_{G-S}(x)+d_{G-S}\left(u^{*}\right)-k|T|-k-h_{G}\left(S, T^{*}\right) \\
& \leq k|S|+\sum_{x \in T} d_{G-S}(x)-k|T|-\left(h_{G}(S, T)-1\right) \leq \delta_{G}(S, T)+1 .
\end{aligned}
$$

Therefore, $\delta_{G}\left(S^{*}, T^{*}\right) \leq \delta_{G}(S, T)$ by Lemma 2.1(ii), which contradicts the maximum of $|S \bigcup T|$.

Similarly, we can prove that $e_{G}(u, T) \leq k-1$ for each $u \in V(G)-(S \bigcup T)$.

Lemma 2.4 (see [4]). Let $m, n, s, t$, and $\omega_{0}$ be nonnegative integers. Suppose that $m \geq 3$, $\omega_{0} \geq 4$, and $m\left(\omega_{0}-1\right) \leq n-s-t-3$. Then it holds that

$$
m-1+s+t \leq \frac{1}{3}\left[n+2\left(s+t+1-\omega_{0}\right)\right] .
$$

Proof of Theorem 1.4. If $G$ contains a complete bipartite graph $K_{n / 2, n / 2}$ as a subgraph when $n$ is even, then Theorem 1.4 holds by [1, Theorems 8.9 and 8.12]. So we may suppose that $G$ does not contain a complete bipartite graph as a subgraph when $n$ is even.

Suppose that there exists an edge $e$ such that $G-e$ has no $k$-factor. By Lemma 2.2, there exists $S_{0}, T_{0} \subseteq V(G)$ with $S_{0} \cap T_{0}=\varnothing$ such that $\delta_{G}\left(S_{0}, T_{0}\right)<\varepsilon\left(S_{0}, T_{0}\right)$. Clearly $S_{0} \cup T_{0} \neq$ $\varnothing$. Otherwise, $\delta_{G}(\varnothing, \varnothing)<\varepsilon(\varnothing, \varnothing)=0$ implies $\delta_{G}(\varnothing, \varnothing) \leq-2$ by Lemma 2.1(ii) which contradicts the fact that $G$ is 2-connected. Set $\theta=\varepsilon\left(S_{0}, T_{0}\right)$; obviously, $\theta=2$. We choose disjoint subsets $S$ and $T$ of $V(G)$ such that $S$ and $T$ satisfy the condition of Lemma 2.3. It is easy to check that $S \cup T \neq \varnothing$.

By Theorem 1.1 and Lemma 2.1, we have $\delta_{G}(S, T)=0$. Therefore,

$$
\omega \geq k|S|+\sum_{x \in T} d_{G-S}(x)-k|T|
$$

where $\omega$ denotes the number of components in $U:=G \backslash(S \bigcup T)$. 
If $U \neq \varnothing$, let $C_{1}, C_{2}, \ldots, C_{\omega}$ be the components of $U$, labelled in such a way that their orders $m_{1}, m_{2}, \ldots, m_{\omega}$ are nondecreasing. By Lemma 2.3 , we have $m_{j} \geq 3(1 \leq j \leq \omega)$.

Let $s=|S|, t=|T|$. Note that if $U \neq \varnothing$, then

$$
|U|=n-s-t \geq 3 \omega
$$

and

$$
d_{G}(u) \leq m_{j}-1+s+t
$$

for every $u \in C_{j}(1 \leq j \leq \omega)$. In particular, we note that when $\omega \geq 2$,

$$
m_{1} \leq \frac{n-s-t}{\omega}, \quad m_{2} \leq \frac{n-s-t-3}{\omega-1} .
$$

If $T \neq \varnothing$, we define

$$
h_{1}:=\min \left\{d_{G-S}(v) \mid v \in T\right\}
$$

and let $x_{1} \in T$ be a vertex satisfying $d_{G-S}\left(x_{1}\right)=h_{1}$ for which $\left|N_{T}\left[x_{1}\right]\right|$ is as small as possible. Further, if $T \backslash N_{T}\left[x_{1}\right] \neq \varnothing$, we define

$$
h_{2}:=\min \left\{d_{G-S}(v) \mid v \in T \backslash N_{T}\left[x_{1}\right]\right\}
$$

and let $x_{2} \in T \backslash N_{T}\left[x_{1}\right]$ be a vertex satisfying $d_{G-S}\left(x_{2}\right)=h_{2}$. Obviously, we have that

$$
\begin{gathered}
h_{1} \leq h_{2}, \\
d_{G}\left(x_{i}\right) \leq s+h_{i} \quad(i=1,2) .
\end{gathered}
$$

By (2.7), we have that

$$
\omega \geq k s+\left(h_{1}-k\right)\left|N_{T}\left[x_{1}\right]\right|+\left(h_{2}-k\right)\left|T \backslash N_{T}\left[x_{1}\right]\right| .
$$

We need to find a pair of nonadjacent vertices $u, v$ in $G$ such that

$$
\max \left(d_{G}(u), d_{G}(v)\right)<\frac{n}{2}
$$

In fact, it suffices to prove that at least one of the following three statements holds in each case.

(A) $\omega \geq 2$ and $m_{2}-1+s+t<n / 2$. (Then (2.16) holds for any $u \in V\left(C_{1}\right), v \in V\left(C_{2}\right)$, by $(2.9)$ and $m_{1} \leq m_{2}$.)

(B) $T \neq \varnothing, \omega>0, d_{G}\left(x_{1}\right)<n / 2$, there exists a vertex $u \in V(U) \backslash N\left(x_{1}\right)$ such that $d_{G}(u)<n / 2$. (Then (2.16) holds with $u=u$ and $v=x_{1}$.)

(C) $T \neq \varnothing, T \backslash N_{T}\left[x_{1}\right] \neq \varnothing$, and $s+h_{2}<n / 2$. (Then (2.16) holds with $u=x_{1}$ and $v=x_{2}$, by $(2.14)$ and $h_{1} \leq h_{2}$.) 
Case $1(T=\varnothing)$. Then $s \geq 1$ since $S \cup T \neq \varnothing$. We have $\omega \geq k s \geq 3 s \geq 3$ by (2.7). So $s \geq 2$ and $\omega \geq 3 s>4$. Otherwise, when $s=1$, it holds that $\omega \geq 3$, which contradicts the fact that $G$ is 2 -connected. By (2.7) and (2.8), we get $s \leq n /(3 k+1)$. This inequality, together with (2.10), gives

$$
\begin{aligned}
m_{2}-1+s+t & \leq \frac{n-s-3}{\omega-1}-1+\frac{n}{3 k+1} \\
& \leq \frac{n-5}{2 k-1}-1+\frac{n}{3 k+1}<\frac{n}{2}
\end{aligned}
$$

This shows (A) in this case.

Therefore we may assume $T \neq \varnothing$.

Case $2(T \neq \varnothing)$.

Case $2.1\left(h_{1} \geq k+2\right)$. Let

$$
\omega_{0}:=k s+\left(h_{1}-k\right) t
$$

When $s=0$ and $t=1$, we have $\omega \geq \omega_{0} \geq 2$, which contradicts that $G$ is 2 -connected. Suppose that $s \neq 0$ or $t \geq 2$, then $\omega_{0} \geq 4$, and so, by Lemma 2.4, with $m=m_{2}$,

$$
\begin{aligned}
m_{2}-1+s+t & \leq \frac{1}{3}\left[n+2\left(s+t+1-k s-h_{1} t+k t\right)\right] \\
& =\frac{1}{3}\left[n-2(k-1) s-2\left(h_{1}-k-1\right)+2\right]<\frac{n}{3},
\end{aligned}
$$

which shows that $(\mathrm{A})$ holds in this case.

Case $2.2\left(h_{1}=k+1\right)$. Let $\omega_{0}:=k s+\left(h_{1}-k\right) t$, and suppose that $s \neq 0$ or $t \geq 4$. Then $\omega \geq \omega_{0} \geq 4$, using the same arguments as in Case 2.1, we get that

$$
m_{2}-1+s+t<\frac{n+2}{3}<\frac{n}{2} .
$$

This also shows (A) in this case. Thus we may consider the following three cases.

(i) $s=0$ and $t=1$.

Clearly, $\delta_{G}(S, T)=0=\epsilon(S, T)$. According to the choice of $S$ and $T$, when $T \neq \varnothing$ and $|S \cup T| \geq 2$, we have $\delta_{G}(S, T) \geq 2$, which is a contradiction by Lemma 2.2.

(ii) $s=0$ and $t=3$.

Then by (2.7) we have $\omega \geq \omega_{0} \geq 3$. By (2.14), we have

$$
m_{2}-1+s+t \leq \frac{n-s-t-3}{\omega-1}-1+s+t \leq \frac{n-6}{2}-1+3<\frac{n}{2},
$$

which shows that $(\mathrm{A})$ holds in this case.

(iii) $s=0$ and $t=2$.

Let $T=\left\{y_{1}, y_{2}\right\}$, and $d_{G}\left(y_{i}\right)=k+1$, for $i=1,2$. Otherwise, $\omega \geq 3$ since $\delta(G) \geq k+1$. We prove in this case that (A) holds in a similar way to that in (ii). Since $k \geq 3$ and $n \geq 4 k+1$, we have that $k-1<n / 2$. So, we may suppose that $y_{1}$ and $y_{2}$ are adjacent, otherwise, (2.16) holds with $u=y_{1}$ and $v=y_{2}$. Since $G$ is 2-connected and $\omega \geq \omega_{0}=2$, we may assume that $y_{1}$ is adjacent to some vertex of a component, say $C_{2}$, where $C_{1}, C_{2}, \ldots, C_{\omega}$ are the components of $G-(S \cup T)$. Thus we have the following claim. 
Claim 1. $y_{1}$ can be adjacent to at most $k-1$ vertices of $C_{1}$.

Note that $m_{1} \leq(n-2) / 2$. Suppose that $m_{1}=(n-2) / 2$. Since $(n-2) / 2 \geq k+1$, there exists at least one vertex $u_{1}$ of $V\left(C_{1}\right)$ not adjacent to $y_{1}$, and $d_{G}\left(u_{1}\right) \leq m_{1}-1+1<n / 2$. Thus (2.16) holds with $u=u_{1}$ and $v=y_{1}$. Thus we assume that $m_{1}<(n-2) / 2$. Clearly, $m_{1} \geq k$ since $\delta(G) \geq k+1$. Note that $d_{G}(u) \leq m_{1}-1+2<n / 2$ for every $u \in V\left(C_{1}\right)$. By Claim 1, there exists at least $u_{2} \in V\left(C_{1}\right)$ not adjacent to $y_{1}$. Thus (2.16) holds with $u=u_{2}$ and $v=y_{1}$.

For the case where $0 \leq h_{1} \leq k$, since $\min \left\{d_{G}(u) \mid u \in V(G)\right\} \geq k+1$ by the hypothesis of the theorem, it holds that

$$
s \geq k-h_{1}+1
$$

We will prove that $d_{G}\left(x_{1}\right)<n / 2$ in the case where $0 \leq h_{1} \leq k$.

Case $A\left(h_{1}=0\right)$. By $(2.7)$, we have $0 \geq k s-k t-\omega, G[T]$ is an isolated set if the equality holds. By (2.8), we have $k s-k t-\omega \geq k s-k(n-s)$, and $n-s-t=\omega=0$ when the equality holds. Thus,

$$
0 \geq k s-k t-\omega \geq k s-k(n-s) .
$$

It follows from the inequality above that $G[T]$ is an isolated set and $n-s-t=\omega=0$ if none of the inequalities in (2.23) is strict. Moreover, in this case, we have $s=t=n / 2$. From (2.14) we get

$$
d_{G}\left(x_{1}\right) \leq h_{1}+s=\frac{n}{2} .
$$

If $d_{G}\left(x_{1}\right)=n / 2$, it is easy to see that each vertex in $T$ is adjacent to all vertices in $S$ by the choice of $x_{1}$. Therefore, $G$ contains $K_{n / 2, n / 2}$ as a subgraph, which is a contradiction. So $d_{G}\left(x_{1}\right)<n / 2$.

If one of the inequalities in (2.23) is strict, we can get $s<n / 2$ from $(2.23)$, thus $d_{G}\left(x_{1}\right) \leq$ $s+h_{1}<n / 2$ by (2.14).

Case $B\left(h_{1}=1\right)$. In this case, it follows from (2.7) and (2.8) that

$$
0 \geq k s+(1-k) t-\omega \geq k s+(1-k)(n-s) .
$$

Thus by (2.14) we have that

$$
d_{G}\left(x_{1}\right) \leq h_{1}+s \leq 1+\frac{(k-1) n}{2 k-1}<\frac{n}{2} .
$$

Case $C\left(2 \leq h_{1} \leq k-1\right)$. It follows from (2.7) And (2.8) that

$$
0 \geq k s+\left(h_{1}-k\right) t-\omega \geq k s+\left(h_{1}-k\right)(n-s),
$$

thus $s \leq n-k n /\left(2 k-h_{1}\right)$. Suppose that $d_{G}\left(x_{1}\right) \geq n / 2$, by $(2.14)$, we have that

$$
\frac{n}{2} \leq s+h_{1} \leq n-\frac{k n}{2 k-h_{1}}+h_{1} .
$$

So $n \leq 4 k-2 h_{1} \leq 4 k-4$, which contradicts the fact that $n \geq 4 k+1$. 
Case $D\left(h_{1}=k\right)$. Thus $s \geq 1$ by (2.22). From (2.7) we have that $\omega \geq k s$. Suppose that $d_{G}\left(x_{1}\right) \geq n / 2$, by (2.14), we have that

$$
k s \geq k+s-1 \geq d_{G}\left(x_{1}\right)-1 \geq \frac{n-2}{2} .
$$

Thus, by (2.8), we have that

$$
n-s-t \geq 3 \omega \geq 3 k s \geq \frac{3(n-2)}{2},
$$

which is a contradiction.

Case $3\left(0 \leq h_{1} \leq k\right.$ and $\left.T=N_{T}\left[x_{1}\right]\right)$. In this case $t \leq k$ unless $h_{1}=k$. Thus it follows from (2.7) and (2.22) that

$$
\omega \geq k s+\left(h_{1}-k\right) t \geq k+\left(k-h_{1}\right)(k-t) \geq k \geq 3 .
$$

Suppose that $V\left(C_{j}\right) \subset N_{G}\left(x_{1}\right)$ for some $j(1 \leq j \leq \omega)$. Since $|T|=\left|N_{T}\left(x_{1}\right)\right|+1$ and $\left|V\left(C_{j}\right)\right| \leq e\left(x_{1}, U\right)$, we get

$$
d_{G / S}(u) \leq|T|+\left(\left|V\left(C_{j}\right)\right|-1\right) \leq\left|N_{T}\left(x_{1}\right)\right|+e\left(x_{1}, U\right)=d_{G / S}\left(x_{1}\right)=h_{1} \leq k
$$

for every $u \in C_{j}$, which contradicts the result of Lemma 2.3. Hence $V\left(C_{j}\right) \not \subset N_{G}\left(x_{1}\right)$, and so there exists a vertex $u \in C_{j}$, which is not adjacent to $x_{1}$.

Let $u_{1} \in V\left(C_{1}\right) \backslash N_{G}\left(x_{1}\right)$. If $d_{G}\left(u_{1}\right)<n / 2$, then (B) holds. Thus we may assume that $d_{G}\left(u_{1}\right) \geq n / 2$. Note that $d_{G}\left(u_{1}\right)$ is strictly less than the upper bound in (2.9) because $u_{1}$ is not adjacent to all vertices of $T$. Therefore, we obtain

$$
\frac{n}{2} \leq d_{G}\left(u_{1}\right) \leq m_{1}-1+s+(t-1) \leq \frac{n-s-t}{3}+s+t-2 .
$$

Hence, it follows that

$$
4 s \geq n-4 t+12
$$

On the other hand, by (2.8) and (2.31), we have that

$$
(3 k+1) s \leq n-\left[3\left(h_{1}-k\right)+1\right] t .
$$

This inequality, together with (2.34), implies that

$$
\begin{aligned}
3(k-1) n & \leq(3 k+1)(4 s+4 t-12)-4 n \\
& \leq 12\left(2 k-h_{1}\right) t-36 k-12 \\
& \leq 12\left(2 k-h_{1}\right)\left(h_{1}+1\right)-36 k-12<12(k-1)^{2},
\end{aligned}
$$

which contradicts that $n \geq 4 k+1$.

Thus we may assume that $T \backslash N_{T}\left[x_{1}\right] \neq \varnothing$. Let $p=\left|N_{T}\left[x_{1}\right]\right|$. We know that $t \geq p+1$, $h_{1} \geq p-1$.

Case $4\left(0 \leq h_{1} \leq k-1\right.$ and $\left.T \backslash N_{T}\left[x_{1}\right] \neq \varnothing\right)$. 
Subcase $4.1\left(h_{1} \leq h_{2} \leq k-1\right)$. Since $\omega \leq n-s-t$ and $k-h_{2} \geq 1$, it follows by (2.15) that

$$
\left(k-h_{2}\right)(n-s-t) \geq \omega \geq k s+\left(h_{1}-k\right) p+\left(h_{2}-k\right)(t-p) .
$$

Therefore

$$
\left(k-h_{2}\right)(n-s)-k s \geq\left(h_{1}-h_{2}\right) p \geq\left(h_{1}-h_{2}\right)\left(h_{1}+1\right) .
$$

Since $p \leq h_{1}+1$ and, by hypothesis, it holds that $n \geq 4 k+1>4 k$, we get that

$$
h_{2} \cdot \frac{n}{2}>h_{2} \cdot 2 k
$$

We may suppose that $s \geq n / 2-h_{2}$, since otherwise (C) holds. So, we have that

$$
\left(s-\frac{n}{2}\right)\left(2 k-h_{2}\right) \geq-h_{2}\left(2 k-h_{2}\right) .
$$

Adding (2.38), (2.39), and (2.40), we obtain

$$
\begin{aligned}
0 & >h_{2}^{2}-h_{2}\left(h_{1}+1\right)+h_{1}^{2}+h_{1} \\
& =\frac{1}{4}\left(2 h_{1}-h_{2}\right)^{2}+\frac{3}{4}\left(h_{2}-\frac{2}{3}\right)^{2}+h_{1}-\frac{1}{3} .
\end{aligned}
$$

For nonnegative integers $h_{1}$ and $h_{2}, h_{2}^{2}-h_{2}\left(h_{1}+1\right)+h_{1}^{2}+h_{1} \geq-1 / 3$ implies that $h_{2}^{2}-$ $h_{2}\left(h_{1}+1\right)+h_{1}^{2}+h_{1} \geq 0$. So, the above inequality is impossible.

For the case where $0 \leq h_{1} \leq k-1$ and $h_{2} \geq k$, since $t \geq p+1$, we have $n-s-t \leq$ $n-s-p-1$. Further, since $h_{2} \geq k$, using (2.8), (2.15) we have

$$
n-s-p-1 \geq n-s-t \geq 3 \omega \geq 3\left[k s+\left(h_{1}-k\right) p\right],
$$

that is,

$$
(3 k+1) s \leq n+\left(3 k-3 h_{1}-1\right) p-1 .
$$

Subcase $4.2\left(h_{2}=k\right)$. Since $3 k-3 h_{1}-1>0$ and $h_{1} \geq p-1$, it follows by $(2.43)$ that

$$
(3 k+1) s \leq n+\left(3 k-3 h_{1}-1\right)\left(h_{1}+1\right)-1 .
$$

By the same reason as in the proof of Subcase 4.1, we may suppose that $s \geq n / 2-h_{2}=$ $n / 2-k$. This inequality, together with (2.44), gives

$$
\begin{aligned}
(3 k-1) n & \leq(3 k+1)(2 s+2 k)-2 n \\
& \leq 2\left[\left(3 k-3 h_{1}-1\right)\left(h_{1}+1\right)-1\right]+6 k^{2}+2 k \\
& =-6 h_{1}^{2}+(6 k-8) h_{1}+6 k^{2}+8 k-4 \\
& <-3 h_{1}^{2}+(6 k-12) h_{1}+6 k^{2}+8 k-2 \\
& =-3\left[h_{1}-k+2\right]^{2}+9 k^{2}-4 k+10 \\
& \leq 9 k^{2}-4 k+10<(3 k+1)(3 k-1),
\end{aligned}
$$

which contradicts that $n \geq 4 k+1$, for $k \geq 3$. 
Subcase $4.3\left(h_{2} \geq k+1\right)$. By (2.15) and (2.22),

$$
\begin{aligned}
\omega & \geq k s+\left(h_{1}-k\right) p+\left(h_{2}-k\right)(t-p) \\
& \geq\left(k-h_{1}\right)(k-p)+t+k-p .
\end{aligned}
$$

Subcase 4.3.1 $(p \leq k-1)$. Let $\omega_{0}=2 k-h_{1}-p+t$. Then $\omega \geq \omega_{0} \geq 5$ by (2.47). Suppose that $m_{2}-1+s+t \geq n / 2$, since otherwise (A) holds. Hence, by (2.10), the hypotheses of Lemma 2.4 are satisfied for $m=m_{2}$. Therefore

$$
m_{2}-1+s+t \leq \frac{1}{3}\left[n+2\left(s+t+1-2 k+h_{1}+p-t\right)\right] .
$$

This, together with the inequality $m_{2}-1+s+t \geq n / 2$, gives

$$
n \leq 4\left(s+h_{1}+p-2 k\right)+4 \text {. }
$$

By (2.43) and (2.49), we obtain

$$
\begin{aligned}
3(k-1) n & \leq(3 k+1)\left[4 s+4 h_{1}+4 p-8 k+4\right]-4 n \\
& \leq 4\left[\left(3 k-3 h_{1}-1\right) p-1\right]+(3 k+1)\left(4 h_{1}+4 p-8 k+4\right) \\
& \leq 4\left(3 k-3 h_{1}-1\right)(k-1)+(3 k+1)\left(4 h_{1}-4 k\right)-4 \leq-4 k-16 .
\end{aligned}
$$

This is obviously impossible.

Subcase 4.3.2 $(p=k)$. In this case $h_{1}=k-1$. Then by (2.22), $s \geq 2$. Since $t \geq p+1=$ $k+1 \geq 4$, by (2.46), we have $\omega \geq k s+t-2 k \geq t$. Let $\omega_{0}=t$, then $\omega \geq \omega_{0} \geq 4$ by (2.46). Hence, by (2.10), the hypotheses of Lemma 2.4 are satisfied for $m=m_{2}$. Therefore,

$$
m_{2}-1+s+t \leq \frac{n+2 s+2}{3} .
$$

By the same reason as in the proof of Subcase 4.3.1, we may suppose that $m_{2}-1+s+$ $t \geq n / 2$. This, together with (2.51), gives

$$
n \leq 4 s+4
$$

Further, when $p=k$ and $h_{1}=k-1,(2.43)$ is as follows:

$$
(3 k+1) s \leq n+2 k-1 .
$$

By (2.52) and (2.53), we get

$$
(3 k+1) s \leq 4 s+2 k+3
$$

which contradicts that $s \geq 2$ in this case.

Case $5\left(h_{1}=k\right.$ and $\left.T \backslash N_{T}\left[x_{1}\right] \neq \varnothing\right)$.

Subcase $5.1\left(k \leq h_{2} \leq k+1\right)$. In this case $t \geq 2$, so that $n-2-s \geq n-s-t$. Since $h_{1}=k$ and $t \geq p+1$, we have

$$
\left(h_{1}-k\right) p+\left(h_{2}-k\right)(t-p) \geq h_{2}-k \text {. }
$$


872 The existence of $k$-factors with prescribed properties

By (2.8) and (2.46), we get

$$
n-s-2 \geq n-s-t \geq 3\left[k s+\left(h_{2}-k\right)\right],
$$

that is,

$$
s \leq \frac{n-2+3\left(k-h_{2}\right)}{3 k+1} \leq \frac{n-2}{3 k+1} .
$$

We still suppose that $s+h_{2} \geq n / 2$, since otherwise (C) holds. Therefore, this, together with (2.57), gives

$$
\frac{n}{2} \leq s+h_{2} \leq \frac{n-2}{3 k+1}+k+1
$$

that is,

$$
(3 k-1) n \leq 6 k^{2}+8 k-2<\left(2 k+\frac{11}{3}\right)(3 k-1),
$$

which contradicts that $n \geq 4 k+1$, for $k \geq 3$.

Subcase $5.2\left(h_{2} \geq k+2\right)$. By (2.22), we have $s \geq 1$. Since $t \geq p+1$ and $p \leq h_{1}+1=k+1$, by (2.46), we get $\omega \geq k s+2(t-p)$. Let $\omega_{0}=k s+2(t-p)$, then $\omega \geq \omega_{0} \geq 5$. By (2.9), the hypotheses of Lemma 2.4 are satisfied for $m=m_{2}$, we get

$$
\begin{aligned}
m_{2}-1+s+t & \leq \frac{1}{3}[n+2(s+t+1-k s-2 t+2 p)] \\
& \leq \frac{1}{3}[n-2(k-1) s-2(p+1)+2+4 p] \\
& \leq \frac{1}{3}[n-2(k-1) s+2(k+1)] \leq \frac{n+4}{3}<\frac{n}{2} .
\end{aligned}
$$

This shows that (A) holds in this case. This completes the proof of Theorem 1.4.

\section{Sharpness of Theorem 1.4}

The condition $\delta(G) \geq k+1$ in Theorem 1.4 is necessary. The assumption that $G$ is 2 connected and $n \geq 4 k+1$ in Theorem 1.4 cannot be weakened any further. Let $k$ be an odd integer such that $k \geq 3$, and let $n$ be an even integer such that $n \geq 4 k+1$. $G_{1}$ is a graph obtained by adding an edge $e$ to connect $K_{k+2}$ and $K_{n-k-2}$. Then $G_{1}$ satisfies all the conditions of Theorem 1.4 except that $G_{1}$ is 1 -connected and $G_{1}$ has no $k$-factors excluding edge $e$. Let $G_{2}=K_{2 k-1}+\left(K_{1} \cup k K_{2}\right)$, then $G_{2}$ satisfies all the conditions of Theorem 1.4 except $n=4 k$. Setting $S=V\left(K_{2 k-1}\right)$ and $T=V\left(K_{1} \cup k K_{2}\right)$, we have $\delta_{G}(S, T)=0<\varepsilon(S, T)=2$; by Lemma 2.2, Theorem 1.4 does not hold.

\section{Acknowledgment}

I would like to thank the referees for their valuable comments which led to a considerable improvement of the original paper. 


\section{References}

[1] G. Chartrand and L. Lesniak, Graphs and Digraphs, The Wadsworth \& Brooks/Cole Mathematics Series, Wadsworth \& Brooks/Cole Advanced Books \& Software, California, 1986.

[2] Y. Egawa and H. Enomoto, Sufficient conditions for the existence of $k$-factors, Recent Studies in Graph Theory (V. R. Kulli, ed.), Vishwa International Publications, India, 1989, pp. 96-105.

[3] T. Iida and T. Nishimura, An ore-type condition for the existence of $k$-factors in graphs, Graphs Combin. 7 (1991), no. 4, 353-361.

[4] T. Nishimura, A degree condition for the existence of $k$-factors, J. Graph Theory 16 (1992), no. 2, $141-151$.

[5] W. T. Tutte, The factors of graphs, Canad. J. Math. 4 (1952), 314-328.

Changping Wang: Department of Mathematics and Statistics, Dalhousie University, Halifax, NS, Canada B3H 3J5

E-mail address: cwang@mathstat.dal.ca 


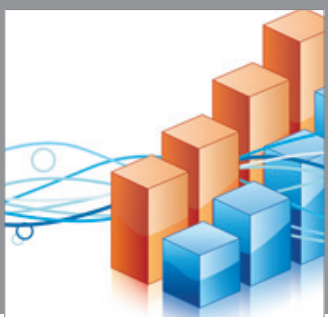

Advances in

Operations Research

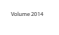

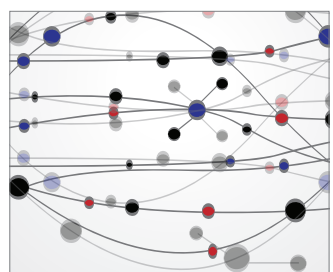

\section{The Scientific} World Journal
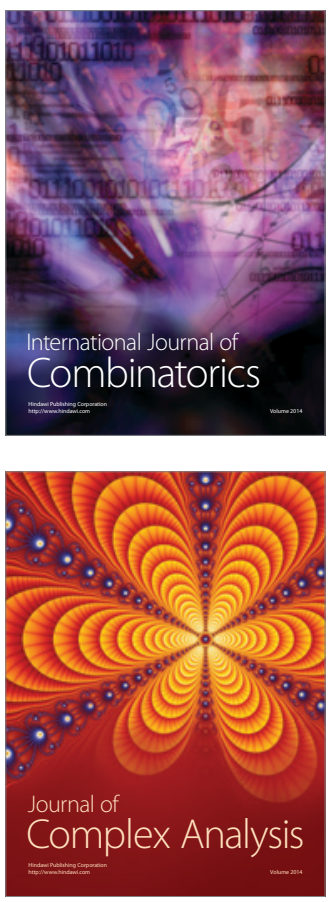

International Journal of

Mathematics and

Mathematical

Sciences
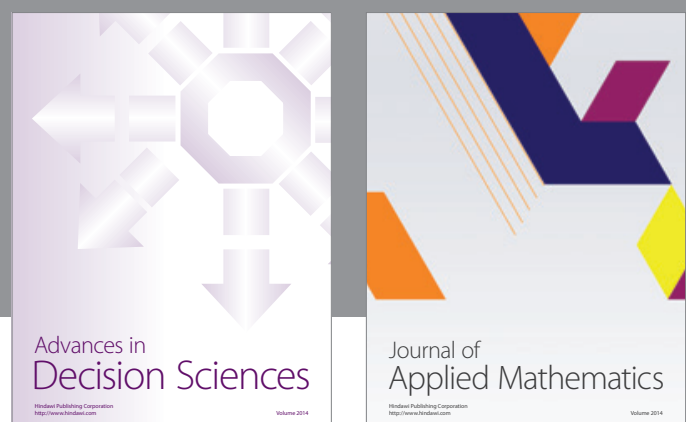

Journal of

Applied Mathematics
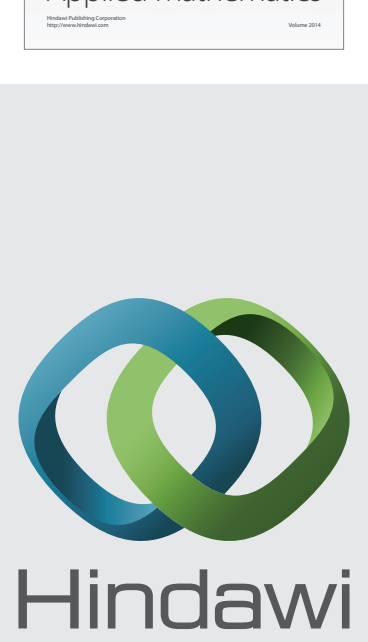

Submit your manuscripts at http://www.hindawi.com
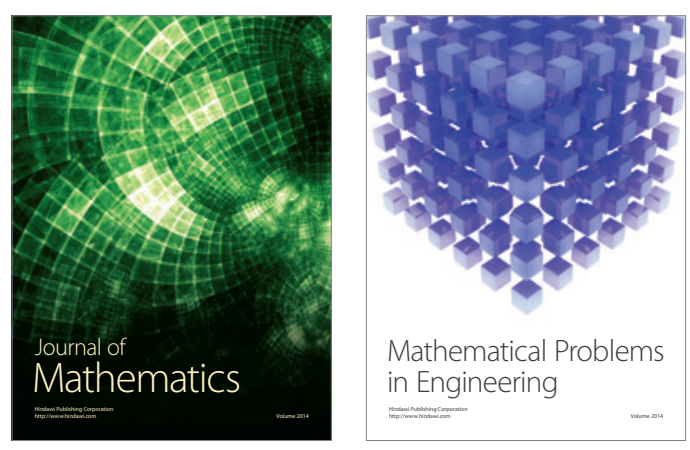

Mathematical Problems in Engineering
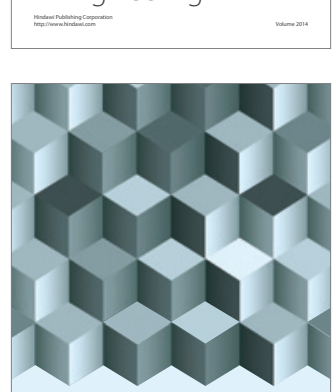

Journal of

Function Spaces
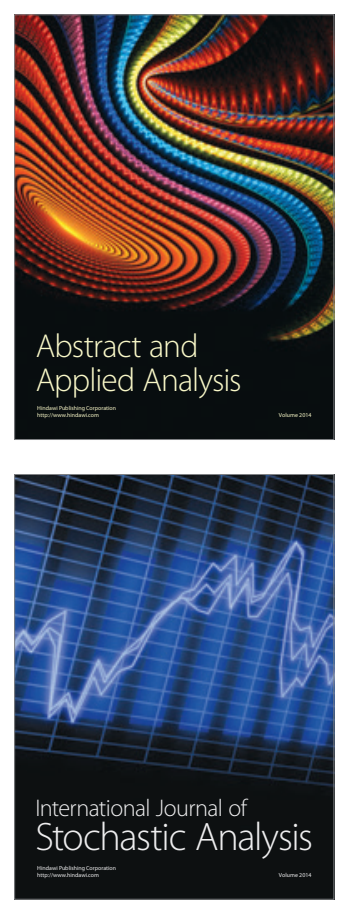

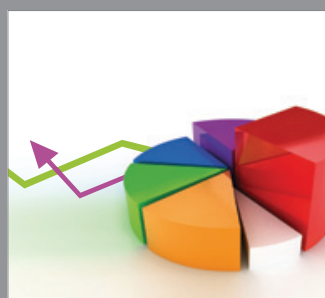

ournal of

Probability and Statistics

Promensencen
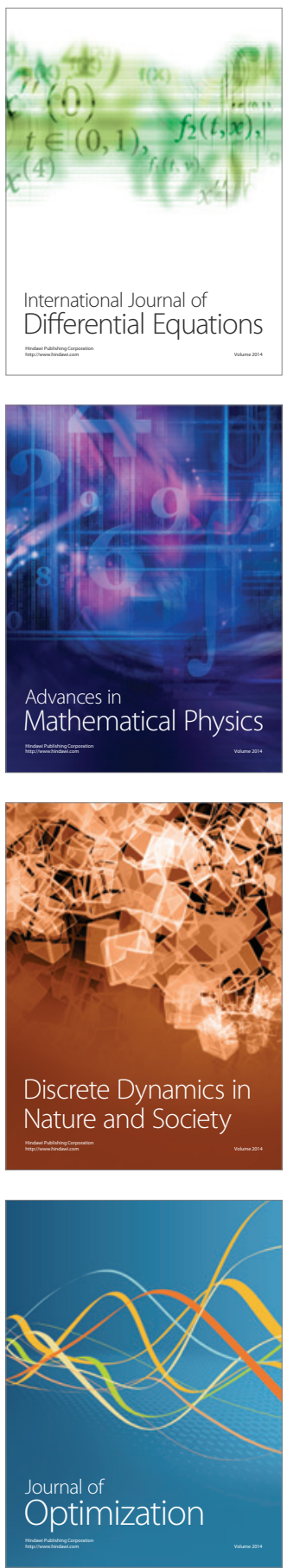